\title{
Dynamic DOE for porthole die extrusion optimisation
}

\author{
Claudio Ciancio ${ }^{1}$, Francesco Gagliardi ${ }^{1}$, Giuseppina Ambrogio ${ }^{1}$ and Luigi Filice ${ }^{1}$ \\ ${ }^{1}$ Department of Mechanical, Energetic and Management Engineering (DIMEG), University of Calabria, 87036 Rende, Italy
}

\begin{abstract}
Extruded profiles are widely used but their design is still difficult. Several parameters have to be considered. This is particularly true when complex geometries, i.e. non-symmetric or hollow profiles have to be obtained. A novel metamodel technique was proposed to define the relationship between input and output process parameters in porthole die extrusion. An iterative dynamic DOE was finally used to improve the accuracy of the model around the most promising area of the process domain.
\end{abstract}

\section{Introduction}

Nowadays, complex profiles made of lightweight materials, such as aluminium or magnesium alloys, are produced largely by extrusion processes [1]. In particular, the extrusion with a porthole die is the most widespread manufacturing process as it allows to manufacture hollow profiles with complex shapes [2,3]. In porthole die extrusion, the extruded billet is split by some bridges which are designed properly to link the mandrel to the die walls. The material streams, crossing the porthole, get inside the so called welding chamber where they join if suitable pressure and temperature conditions are guaranteed [4]. A good weld quality has to be ensured to avoid failed extruded with a careful die design [5]. After the streams welding, the material flows through the bearing orifice, obtaining its final shape. In order to have undistorted profiles, the local lengths of the bearing can be variable along the exit perimeter [6]. Moreover, the bearing length has to be set taking into account grain growth [7] and surface defects [8,9].

Sometimes, it is also helpful to introduce an additional chamber, called pocket, between the welding chamber and the bearing, which section plays an important role in the flow optimization [10,11]. On the other side, the pocket introduction brings to higher extrusion loads [12]. Therefore, a right combination of the bearing land and pocket design has to be defined properly.

The optimization complexity increases if sections with non-symmetric shape have to be produced. According to that, the use of numerical tools is always more widespread and commercial finite element software have been developed to investigate the forming phases $[13,14]$. However, the problem complexity is so high that the exclusive use of finite element model (FEM) can result poorly effective being the numerical approach "case dependent"; on the contrary, the combined use of numerical tools and mathematical regression models can allow defining wider results.

According to that, this paper aims at investigating the influence of geometrical parameters (i.e. bearing length and pocket geometry) to produce undistorted profiles. To make the example more complex and interesting from an industrial point of view, dies with unsymmetrical flow have been considered. More in detail, different die geometries characterised by changing velocity flow at the exit of the die have been tested; both the flow velocity along the profile and the steady-state ram load are measured as response factors. As concerns the former one, it is required that velocity gradient along the exit profile converges to zero for ensuring an homogeneous material flow and preventing the profile distortion; at the same time, the steady-state ram load has to be minimized for limiting the die wear and the required press capacity. The analysis has been based on numerical simulations carried out by the commercial code, Altair HyperXtrude (V-13), to generate the base of knowledge; subsequently, dynamic sampling techniques have been used to build a metamodel starting by a previous model designed for a similar problem as a reference point. All the details are reported in the following sections.

\section{Methodology}

Statistical methods have been used widely in manufacturing to ensure quality and robust process design [15]. Identification of input-output relationships of manufacturing processes is usually a complex task, which involves integration of physical-driven (or even simulation-driven) experiments and data mining algorithms. This task is costly and time consuming. Many approaches have been proposed to apply mathematical methods, techniques and paradigms to the solution of

\footnotetext{
${ }^{\mathrm{a}}$ Corresponding author: claudio.ciancio@unical.it
} 
manufacturing problems. Mathematical model can in fact be used to understand how to improve efficiently a given system configuration analyzing and identifying the factors that affect majorly the investigated responses [16]. In this chapter a new methodology has been presented to perform a faster identification of these relationships by integrating response surface models and similarity functions. The proposed approach allows generating intelligent decision support systems that can be used to study and manage complex manufacturing processes. The system learns basing on accumulated data and adapts itself during the analysis. Therefore, the proposed methodology can be a helpful tool to reduce the cost (time and experiments) and to perform the design of a reengineered process. The aim of the proposed methodology is to identify the relationship that links a set of controlled process parameters and a set of output responses; this one reflects the critical aspects of the manufacturing system. More formally, a transfer function is defined for each output $y_{j} \in Y, y_{j}=f_{j}^{k}\left(x_{1}, x_{2}, \ldots, x_{n}\right)$, where $n$ is the number of controlled parameters, $X=\left\{x_{1}, x_{2}, \ldots x_{n}\right\}$. The transfer function $y_{j}$ approximates the process behavior under a certain process condition $k$. The proposed technique is used to estimate a new model $f_{j}^{k \prime}$ if a new process condition $k^{\prime}$ has to be investigated. A set of data is usually collected and used to train and validate a machine learning model. However, a different approach has been used to reduce the total number of experiments adding the necessary feedbacks with a model (previously defined and used to map the process before the process reengineering) and a sequential sampling procedure (Figure 1). The sequential approach, moreover, offers the possibility to achieve different "milestone" during the learning phase identifying the magnitude of the effect of the analyzed parameters with a small dataset. Other data can be collected subsequently to achieve the required accuracy.

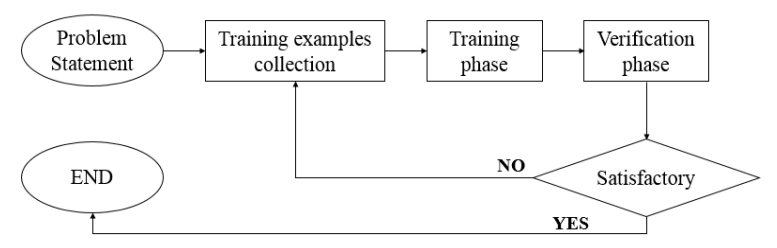

Figure 1. Mathematical approach to define input-output relationships.

The proposed approach allows obtaining the following benefits compared to benchmark methods: 1) ability to map complex shapes using simple transformations (overfitting risk reduction); 2) use of historical data on similar problems; 3) greater flexibility given by the integration between the learning algorithm and the experimental design. The proposed methodology has been performed through the following steps:

- $\quad$ sampling strategy (Phase 1)

- $\quad$ surrogate model preliminary analysis

- surrogate model

- $\quad$ sampling strategy (Phase 2)

- $\quad$ stopping criteria

\subsection{Sampling strategy (Phase 1)}

The sampling strategy used to train the surrogate model is composed by two phases. In the first phase, a small set of data is collected using a benchmark approach to capture the first indications about the influence of each controlled parameters on each output response. In this study, the data are selected using a latin hypercube design [17].

\subsection{Surrogate model preliminary analysis}

A preliminary analysis has been used to identify the complexity of the function to approximate. This phase has been performed to introduce a regularization parameter (or a penalty) to avoid over-fitting. Given a model $f_{j}^{k}$ so that one output component $y_{j}$ of a problem $k$ can be predicted through the equation $y_{j}=f_{j}^{k}\left(x_{1}, x_{2}, \ldots, x_{n}\right)$, the optimal value of a vector has been identified:

$$
\beta=\left\{\beta_{0}, \beta_{1}, \ldots, \beta_{n+1}\right\}
$$

that allow minimizing the error obtained on a second problem, $k^{\prime}$ using a model $f_{j}^{k \prime}$ defined as follows:

$$
f_{j}^{k \prime}=\beta_{0} f_{1}\left(x_{1}+\beta_{1}, x_{2}+\beta_{2}, \ldots, x_{n}+\beta_{n}\right)+\beta_{n+1}(2)
$$

The error $E_{0}$ obtained on the training data by this model will be used as an input parameter to define a risk coefficient.

\subsection{Surrogate model}

In this phase, an alternative and general theory of learning with similarity functions has been developed. The idea of the proposed algorithm is based on the computation of a linear regression function in a high dimensional feature space where the input data are mapped via a nonlinear function. In the nonlinear setting, therefore, the optimization problem corresponds to finding the flattest function in the feature space. The methodology developed in this section can be considered as a generalization of the kernel method [18]. The theory behind kernel functions is based on the fact that many standard algorithms, such as SVR and Perceptron algorithm [19], can be written in such a way that the only way they interact with their data is via computing dotproducts on pairs of examples. Kernel functions are usually used to fit a set of data (training data) trying to build a model that can be generalized for a second set of data (validation data). However, the problem addressed in this phase is different. Given two problems $k$ and $k^{\prime}$ for which two sets of data are available, a surrogate model $f_{j}^{k^{\prime}}$ has been defined through a transformation (denoted henceforth as similarity transformation) on a second model $f_{j}^{k}$ previously defined. It has been denoted as $X_{t}^{k}$ the $\mathrm{t}$-th datum available for the process condition $k$ and by $x_{i, t}^{k}$ the value of the i-th input of $X_{t}^{k}$. The proposed approach has been used as the one which allows obtaining the following benefits compared to classic kernel methods: 
- ability to map complex shapes using simple transformations (overfitting risk reduction);

- use of historical data on similar problems.

A similarity function can be considered as a function that is estimated from the data, or that is chosen to fit the data. However, the similarity function should be as simple as possible to reduce the overfitting risk. Simple similarity transformations can be built considering a weighted Euclidean distance:

$$
d_{w}\left(X_{t}^{k}, X_{p}^{k \prime}\right)=\sqrt{\sum_{i=1}^{n} w_{i}\left(x_{i, t}^{k}-x_{i, p}^{k^{\prime}}\right)^{2}}
$$

where $w_{i}$ is a weight assigned to the $\mathrm{i}$-th process parameter. The similarity function $s_{w}$ is expected to increase decreasing the value $d_{w}$ reaching the value 1 for $d_{w}=0$. So typical similarity function can be:

- Exponential function: $s_{w}=e^{-d_{w}+d_{0}}$

- Linear function: $s_{w}=\frac{1}{1+d_{w}+d_{0}}$

- Logarithmic function: $s_{w}=\frac{1}{\log \left(1+d_{w}+d_{0}\right)+1}$

- Logarithmic function: $s_{w}=\cos \left(d_{w}+d_{0}\right)$

where $d_{0}$ is used to map translational changes of the response surface. The output of a new point $X_{p}^{k \prime}$ is estimated trough the following equation:

$$
y_{j, p}^{k \prime}=\sum_{t=1}^{T} \alpha_{t} s_{w}\left(X_{t}^{k}, X_{p}^{k \prime}\right) y_{j, t}^{k}
$$

where the optimal value of the vector $\alpha=\left\{\alpha_{1}, \alpha_{2}, \ldots, \alpha_{T}\right\}$ is determined through a mathematical model. This one is solved by means of genetic algorithms where it is minimized:

$$
\min \lambda E+\sum_{t=1}^{T} \alpha_{t}^{2}
$$

The parameter $\lambda$ is inversely proportional to the risk coefficient $E_{0}$ determined in section 2.2. Moreover $E_{0}$ is used to tune a parameter $\alpha_{\max }$ that determines the maximum number of variables which can assume any value in addition to 0 .

\subsection{Sampling strategy (Phase 2)}

The similarity function technique has been used to update the response surface of the problem defining a new fitting model. However, the prediction abilities of the new tool could be not yet satisfactory due to the lack of data in some area of the process domain. A new set of experiments has been defined to reduce the inaccuracy of the tool. Each new experiment has been designed using the results of the current metamodel until the accuracy of this tool can be considered statistically satisfactory. More in particular, at each iteration, a set of new experiments is performed considering new samples localized in the so called $\epsilon$-tube. The $\epsilon$-tube is composed by all the possible solution

$$
X_{\epsilon}^{k^{\prime}} \in \Omega:\left|P\left(x \in \Omega^{\mathrm{k}^{\prime}}\right)-P\left(x \notin \Omega^{\mathrm{k} \prime}\right)\right| \leq 2 \epsilon
$$

where $\Omega^{\mathrm{k} \prime}$ is a promising area of the process domain that satisfies some quality constraints.

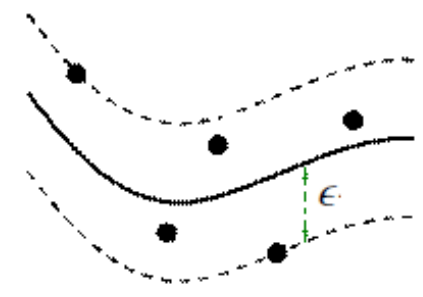

Figure 2. $\epsilon$-tube schematization.

The new set of points to analyze has been selected trying to maximize the distance between the new point and the previous training data. The $\epsilon$ value has been reduced gradually during the iterations. The motivation of this choice is related to the fact that the accuracy of the prediction increases gradually so the subsequent iterations lead to small changes. The proposed approach owns two degrees of freedom that allows reducing the number of required experiments compared to benchmark approaches:

- Parameters weighting: the interaction with the surrogate model allows to identify the most relevant process parameters;

- Data concentration: more experiments are performed in the most promising area of the process domain.

\subsection{Stopping criteria}

According to the preliminary testing, the use of two criteria to stop each iteration of the algorithm has been found profitable, namely:

- the number of experiments performed exceeds a maximum threshold;

- the accuracy of the predictive tool is greater than a given threshold $\varepsilon_{\min }$.

\section{The case study}

Feasibility of asymmetric profile by porthole die extrusion is guaranteed homogenising the material flow. This aim can be pursued by playing with two variables: the bearing length and the pocket geometry. The pocket is a die extension below the welding chamber that is sometime used to get material flow homogenisation. The pocket has to be designed properly taking into account the porthole die geometry in all its parts. According to that, the present research has been focalised on the manufacturing of complex L profile by using a multigates die. This choice has been performed to take also into account the asymmetry between the axis of the extruded billet and the die exit as further variable to consider in the flow homogenization. In detail, the process has been designed for the simultaneous extrusion of two L profiles whose dimensions are $20 \mathrm{~mm}$ and 47 $\mathrm{mm}$ for the short and long length respectively, and $5 \mathrm{~mm}$ for the part thickness (Figure 3.a). The starting billet 
diameter is equal to $146 \mathrm{~mm}$ and the extrusion ratio is therefore of about 27; finally, the billet length is of 150 $\mathrm{mm}$.

Two die configurations that correspond to different material flows into the pocket have been investigated; the distance between billet axis and die gate is the geometric variable (Figure 3.b) which allows the configuration changing.
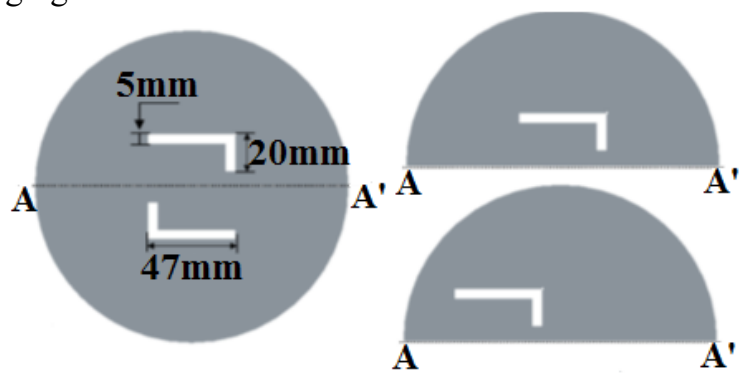

Figure 3. 2D Sketch a) of the investigated geometry and b) of the die configurations.

The parameterized dimensions of the bearing lengths and of the main pocket sizes have been planned and multiplicative constants have been set for changing logically the extrusion die. More in detail, the parameters taken into account are: the local bearing length, $H_{\text {bearing, }}$, the pocket height, $H_{\text {pocket }}$, and the pocket cross section $D_{\text {pocket }}$. Concerning the last investigated factor, this one has been set by starting from the L shape going to widen the section where lower material flow velocity is expected (Figure 4).

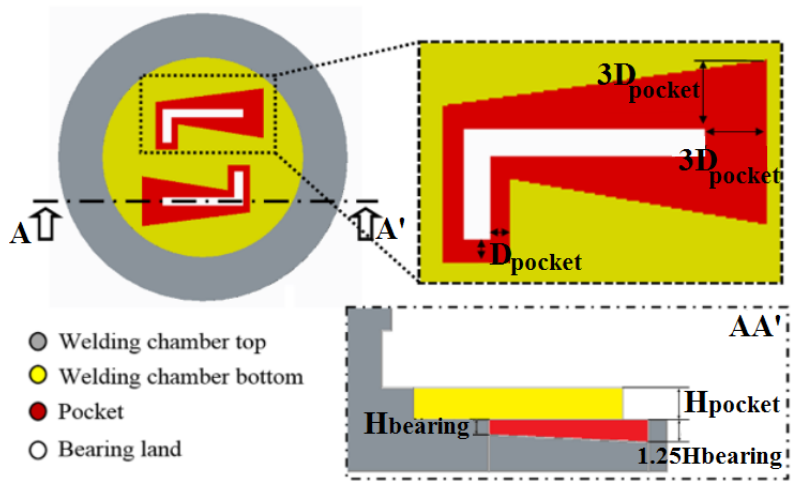

Figure 4. Top and front views of the investigated die.

The proportionality between the lengths of the bearing and the dimensions of the pocket has been established to avoid an unbalanced flow. The multiplicative constants used for varying the investigated variables are summarized in Table 1.

Table 1. Multiplicative constants.

\begin{tabular}{|c|c|c|}
\hline $\begin{array}{c}\mathbf{H}_{\text {bearing }} \\
{[\mathrm{mm}]}\end{array}$ & $\begin{array}{c}\mathbf{H}_{\text {pocket }} \\
{[\mathrm{mm}]}\end{array}$ & $\begin{array}{c}\mathbf{D}_{\text {pocket }} \\
{[\mathrm{mm}]}\end{array}$ \\
\hline 3 & 2.5 & 3 \\
\hline 6 & 5 & 7 \\
\hline 9 & 7.5 & 1 \\
\hline
\end{tabular}

\section{Numerical model}

A reduced number of simulations have been performed for all the process configurations in order to find a general guideline, which can allow the homogeneous distribution of the material flow into the pocket, by properly choosing the pocket geometry and bearing height.

The numerical investigation has been carried out by means of the software Altair HyperXtrude V-13. HyperXtrude is based on an Arbitrary LagrandianEulerian (ALE) approach which is used to get locally material information inside the extrusion die and to predict also the flow of the extruded profile in the bearing region and at its exit.

The numerical model has been designed by considering six elements: the billet, the porthole, the welding chamber, the pocket, the bearing and the finale profile (Figure 5).
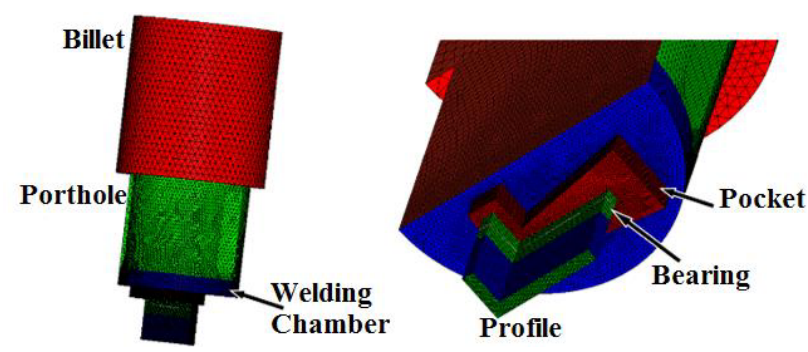

Figure 5. Meshed model.

Half geometry has been simulated by exploiting the symmetry of full model; this choice has been performed to reduce the computational time. For the same reason, the meshed model is characterized by a gradually coarser refinement from the extruded profile to the billet. By doing that, 78000 prismatic elements have been used between bearing and profile; tetra elements have been instead adopted to mesh the remaining zones (about 440000 elements).

The constitutive equation used to describe the aluminium profile behaviour during the extrusion process is the sine hyperbolic law. Regarding the boundary conditions, a ram speed of $1 \mathrm{~mm} / \mathrm{s}$ was utilized; taking into account the extrusion ratio value, the average value expected as profile exit velocity is $27 \mathrm{~mm} / \mathrm{s}$. The initial billet temperature has been set to $500^{\circ} \mathrm{C}$, while the temperatures of the container and the other regions have been set to $450^{\circ} \mathrm{C}$ and $420^{\circ} \mathrm{C}$, respectively. Sticking conditions have been considered at the interface materialdies except of the bearing surface where viscoplastic conditions have been set with a friction coefficient of 0.3 , to consider the material sliding in the orifice [20].

The distribution of the profile velocity at the exit of the bearing zone and the steady state ram load necessary for the extrusion have been the analysed outcomes.

As concerns the profile distortion, this one has been investigated by focusing the attention on the velocity discrepancy between two points on the extruded cross section. The points have been chosen to maximize the difference $(\Delta \mathrm{V})$ in the velocity distribution (Figure 6). 


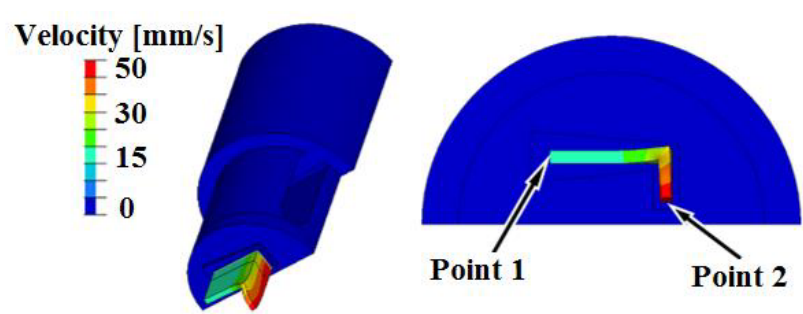

Figure 6. Velocity distribution and detail of the points used for $\Delta \mathrm{V}$ measure.

\section{Discussion of the result}

The optimization procedure described in section 2 has been used to define the most suitable input parameters of a porthole extrusion process. In particular, a polynomial model has been defined to set the relationship between the local bearing length, $H_{\text {bearing }}$, the pocket height, $H_{\text {pocket }}$, the pocket cross section $D_{\text {pocket }}$ and the velocity discrepancy $\Delta \mathrm{V}$ and the ram load $\mathrm{F}$. The proposed approach based on response surface projection has been then used to redefine the model for a different position of the L section. The two objective functions (minimization of the velocity discrepancy and ram load) have been weighted differently and joined into a single objective function:

$$
z=\lambda_{1} \Delta V^{\prime}+\lambda_{2} F^{\prime}
$$

where $\Delta V^{\prime}$ and $F^{\prime}$ are the normalized value of $\Delta V$ and $F$ in the range $0-1$. A value equal to 0.8 is assigned to $\lambda_{1}$ and a value equal to 0.2 to $\lambda_{2}$. The objective function $z$ is therefore also defined in the range $0-1$. The first section has been investigated in a previous study [21] using a full factorial DoE and a second order model for both responses. The input parameters have been investigated inside the range reported in Table 2

Table 2. Design factors information of FEM simulations.

\begin{tabular}{|c|c|c|}
\hline Factor & Unit & Values \\
\hline $\mathrm{H}_{\text {bearing }}$ & $\mathrm{mm}$ & $1.5-3.0-4.5$ \\
\hline $\mathrm{H}_{\text {pocket }}$ & $\mathrm{mm}$ & $3-7-11$ \\
\hline $\mathrm{D}_{\text {pocket }}$ & $\mathrm{mm}$ & $2.5-5-7.5$ \\
\hline
\end{tabular}

The accuracy of the model has been measured through the $\mathrm{R}^{2}$. A value equal to 0.953 has been reached for the $\Delta V$ and a value equal to 0.988 for the $F$. The dynamic sampling and the metamodel based on similarity functions has been then used to analyse the geometry in Figure 3.b.

\subsection{Sampling strategy (Phase 1)}

To start the algorithm it is necessary to select a first set of data to train the model. 9 process configuration have been analyzed using a latin hypercube design sampling. In this stage the selected experiments are not influenced by the model developed for the first geometry.

\subsection{Surrogate Model Preliminary analysis}

The surrogate model for the first section has been used as reference point to develop a new model for the second one not yet investigated using a combination of simple transformations. The only changes allowed in this phase are translations along the $\mathrm{x}$ and $\mathrm{y}$ axes or using $\mathrm{a}$ different magnitude level.

Table 3. Surrogate model (Preliminary analysis)

\begin{tabular}{|c|c|}
\hline Parameter & $\boldsymbol{R}^{\mathbf{2}}$ \\
\hline$\Delta \mathrm{V}$ & 0.769 \\
\hline Ram Load & 0.710 \\
\hline
\end{tabular}

\subsection{Surrogate model}

The results achieved using the preliminary analysis has been used to define the $\lambda$ showed in section 2.3. Different similarity transformations have been tested on this problem trying to map the relationship between the problems analyzed maximizing the value of the available information. The results of this phase are summarized in Table 4:

Table 4. Surrogate model (Preliminary analysis)

\begin{tabular}{|c|c|c|}
\hline Parameter & $\boldsymbol{R}^{\mathbf{2}}$ Phase 1 & Similarity Function \\
\hline$\Delta \mathrm{V}$ & 0.792 & Exponential \\
\hline Ram Load & 0.851 & Exponential \\
\hline
\end{tabular}

As it is possible to see, the best results have been achieved using the exponential function for all the responces. This model has been then used to develop the new DoE strategy.

\subsection{Sampling strategy (Phase 2)}

The results obtained have been utilized to define a second set of experiments. The experiments have been selected iteratively around a $\epsilon$-tube $(\epsilon=0.05)$ until the accuracy has been greater than a predetermined threshold. In this case-study, $\epsilon$-tube has been defined as the portion of the process domain with a predicted value of $z$ less than 0.1 . The $\epsilon$ value has been iteratively modified at each iteration $t$ according to the residual number of maximum experiments available:

$$
\epsilon_{t}=\epsilon_{0}-\epsilon_{0} \frac{N_{t}}{N_{\operatorname{Max}}}
$$

where $N_{t}$ and $N_{\text {Max }}$ are respectively the current and the maximum number of performed experiments.

\subsection{Stopping criteria}

Two stopping criteria have been used in this analysis. The accuracy of the tool has been measured using the $R^{2}$ with a leave-one-out cross validation. The threshold value of this parameter has been fixed equal to 0.97 equal to the average $R^{2}$ on the two responses obtained with the second order model and a full factorial DoE for the first Stackup. The maximum number of experiments has been set 
equal to 27 (dataset size of the full factorial DoE used in the previous analysis).

It is possible to see that the proposed algorithm has reached a superior level of accuracy using the same dataset size. The algorithm has been arrested after 18 experiments with a reduction of $33 \%$ of the dataset size compared to the one used for the first geometry. The value of $\Delta V$ ranges between $0.19-15.75$ while the value of $F$ between 7.134-7.822. The model has been finally used to select an optimal configuration which minimize both objectives.

Table 5. Model accuracy

\begin{tabular}{|l|l|l|}
\cline { 2 - 3 } \multicolumn{1}{c|}{} & Geometry 1 & Geometry 2 \\
\hline DoE & Full Factorial & $\begin{array}{l}\text { Adaptive } \\
\text { sampling }\end{array}$ \\
\hline Methodology & $\begin{array}{l}\text { Polynomial } \\
\text { Model }\end{array}$ & $\begin{array}{l}\text { Similarity } \\
\text { Function }\end{array}$ \\
\hline $\mathrm{N}^{\circ}$ Data & 27 & 18 \\
\hline Accuracy $\Delta V\left(R^{2}\right)$ & 0.953 & 0.968 \\
\hline Accuracy $F\left(R^{2}\right)$ & 0.988 & 0.991 \\
\hline
\end{tabular}

Table 6. Optimal Configuration geometry 2 (input values)

\begin{tabular}{|c|c|}
\hline Factor & Value \\
\hline $\mathrm{H}_{\text {bearing }}$ & $1.50 \mathrm{~mm}$ \\
\hline $\mathrm{H}_{\text {pocket }}$ & $6.72 \mathrm{~mm}$ \\
\hline $\mathrm{D}_{\text {pocket }}$ & $6.93 \mathrm{~mm}$ \\
\hline
\end{tabular}

Table 7. Optimal Configuration geometry 2 (output values)

\begin{tabular}{|c|c|c|}
\hline Parameter & Predicted & Real value \\
\hline$\Delta V$ & 0.431 & 0.700 \\
\hline Ram Force & 7.142 & 7.134 \\
\hline $\mathrm{Z}$ & 0,011 & 0.016 \\
\hline
\end{tabular}

\section{Conclusions}

Porthole die extrusion has been utilized widely for producing lightweight material profiles characterized by complex shapes. Different variables have to be constantly monitored for guaranteeing good product quality; for maintaining these output values inside desirable ranges, several geometric and process parameters (input values) have to be set properly for each investigated geometry. This aspect drastically increases the complexity of the design phase as far as postpones the lead time before a new production rump up.

In the proposed work, an innovative procedure based on the recursive and intelligent use of already known knowledge in generating new one for new process design has been applied to solve the above specified problem.

A new algorithm able to determine automatically input-output relationships in manufacturing applications has been applied for mapping the porthole die extrusion process of complex hollow profiles. In detail, three input and two output variables have been taken into account.

The discussed results demonstrate that the proposed method based on an adaptive sampling DoE and a prediction tool based on shape similarities between a set of surfaces leads to better prediction compared to benchmark approaches used for this kind of problems.
The proposed methodology could be used to solve problems on different domains. However, this technique is particularly effective if at least one of this conditions is satisfied:

- restricted interest in a particular subarea of the solutions space;

- some interactions between input parameters are negligible.

All these conditions are exploited in order to obtain the desired information reducing the number of required data which is directly related to the cost and time necessary to perform the parameter selection for the process start up.

\section{References}

1. G. Liu, J. Zhou, J. Duszczyk, J. of Mat. Proc. Tech. 200/1-3, 185-198 (2008);

2. H. Valberg, Int. J. of Mat. \& Prod. Techn. 17, 497-556 (2002);

3. L. Li, H. Zhang, J. Zhou, J. Duszczyk, G.Y. Li, Z.H. Zhong, Mater. \& Des, 29/6, 1190-1198 (2008);

4. K.J. Kim, C.H. Lee, D.Y. Yang, Jour. of Mater. Proc. Techn., 130-131, 426-431 (2002);

5. J. Liu, G. Lin, D. Feng, Y. Zou, L. Sun, Central South University Technology, 17, 688-696 (2010);

6. R. Mayavaram, U. Sajja, C. Secli, S. Niranjan, Proc. CIRP, 12, 276-281 (2013).

7. F. Gagliardi, T. Citrea, G. Ambrogio and L. Filice, Mater. \& Des. 60, 274-281 (2014);

8. G. Fang, J. Zhou, J. Duszczyk, Jour. of Mater. Proc. Techn., 209/6, 3050-3059 (2009);

9. M. Bauser, K. Siegert, Extrusion: Second Edition, ASM International 242-243 (2006);

10. Y. He, S. Xie, L. Cheng, G. Huang, Y. Fu, Trans of Nonf. Met. Soc. of China, 20/6, 1067-1071 (2010);

11. J. Lof, Developments in finite element simulations of aluminum extrusion, thesis (2000);

12. Q. Li, C.J. Smith, C. Harris, M.R. Jolly, Jour. of Mater. Proc. Techn., 135/2-3, 189-196 (2003);

13. J. Zhou, L. Li, J. Duszczyk, Jour. of Mater. Proc. Techn., 134, 383-397 (2003);

14. A.J. Williams, A.K. Slone, T.N. Croft, M. Cross, Comp. Meth. in Appl. Mech. and Eng, 199/33-36, 2123-2134 (2010);

15. M. J. Anderson, "Response Surface Methods (RSM) Achieve Design for Six Sigma (DFSS) Goals for Medical Device Manufacturing;

16. L. Monostori, Eng. Appl. Artif. Intel. 16.4 (2003): 277-291;

17. M.D. Shields, J. Zhang, Rel. Eng. \& Syst. Saf. 148, 96-108 (2016);

18. D. Basak, S. Pal, D.C. Patranabis, Neur. Inf. Processing-Letters and Reviews, 11.10 203-224 (2007);

19. M. Collins, in Proc. ACL-02 Conf. Emp. Met. in Nat. Lan. Proc. 10, (2002);

20. X. Ma, M.B. de Rooij, D.J. Shipper, Wear, 278-279, 1-8, (2012).

21. T. Citrea, F. Gagliardi, G. Ambrogio, L. Filice, in Proc. XII A.I.Te.M Conf. (2015). 